\title{
An LFT/SDP Approach to the Uncertainty Analysis for State Estimation of Water Distribution Systems
}

\author{
Atulya K. Nagar and Roger S. Powell \\ Department of Systems Engineering, \\ Brunel University, Uxbridge UB8 3PH, U.K. \\ [atulya.nagar, roger.powell]@brunel.ac.uk
}

\begin{abstract}
A state estimator is an algorithm that computes the current state of a time-varying system from on-line measurements. Physical quantities such as measurements and parameters are characterised by uncertainty. Understanding how uncertainty affects the accuracy of state estimates is therefore a prerequisite to the application of such techniques to real systems. In this paper we develop a method of uncertainty analysis based on linear fractional transformations (LFT) and obtain ellipsoid-of-confidence bounds by recasting the LFT problem into a semidefinite programming problem (SDP). The ideas are illustrated by applying them to a simple water distribution network.
\end{abstract}

\section{Introduction}

State estimation is defined as the computation of the minimum set of values necessary to completely describe all other pertinent variables in a given system from some measurement data [19]. When applied to water or gas distribution networks it can be viewed as an on-line monitoring system capable of tracking the time varying flows and pressures in real-time. In power systems, voltages and power flows can be estimated.

The state estimator algorithm maps the available new information (from measurements) into a statespace using an over-determined set of (non-linear) equations. This is typically formulated as a projection resulting in a minimisation problem, e.g. weighted least squares (WLS). In general, static state-estimation can be categorised according to the minimisation cost function used; either a quadratic, e.g. weighted least squares, or a non-quadratic function. A great number of papers on power systems state estimation have concentrated on variations on the WLS theme by including different methods of bad data detection and identification. Examples include identification by elimination [22], hypothesis testing identification [15], 
combinatorial optimisation identification [16] and non-quadratic criteria [13]. Irving et al [14] suggested a method for weighted least absolute values (WLAV) estimation formulated as a linear program that has also been used for water distribution networks [21]. WLAV is normally considered more robust in the presence of bad data because it selects the $n$ 'best' measurements where $n$ is the number of state variables and ignores the remaining measurements. However, WLS is considered to be more robust than WLAV where the data are noisy $[20]$.

Measurement uncertainty is anticipated by the above methods but there has been little work, in this applied field, on the effects of the combination of measurement and parametric uncertainty. Bargiela and Hainsworth [2] introduced the idea of incorporating measurement bounds with the aim of increasing the robustness of state estimation under uncertainty. This idea was developed by Brdys and Chen [4], and Gabrys and Bargiela [8], as the so-called set-bounded state estimation problem (SBSE). In SBSE, the aim is to define only the region in which the solution must lie. As a result, one can obtain upper and lower bounds on all the state variables but not the state estimate itself. The traditional (e.g. WLS) state estimation methods are referred to as point state estimators (PSE), since they produce a point in state-space.

Optimisation criteria such as regularised least squares, ridge regression and total least squares permit, in one way or another, the incorporation of a priori information about the unknown parameters in the problem statement e.g. [12]. In recent years many new algorithms have been proposed to solve the total least squares (TLS) problem of $A x \approx b$ (with uncertainties in $A$ and $b$ ). For example, Chandrasekaran et al [5] formulate a parameter estimation problem in the presence of bounded data uncertainties and solve the optimisation problem to obtain a solution in terms of the unique positive root of a secular equation. Alternatively, the structured total least norm (STLN) formulation and algorithm described in [18] preserves the problem structure for solving problems related to TLS and permits the minimisation of error in different norms.

Doyle [6] introduced the concept of LFTs in order to examine the robustness of dynamic control systems under parametric uncertainty. El Ghaoui and Lebret [11] consider robust least squares (RLS), and structured robust least squares (SRLS) formulations with unknown but bounded data matrices and minimise the worstcase residual error using (convex) semidefinite programming techniques and interior point methods. They also use the linear fractional representation introduced by Doyle [6] to pose the problem as a linear fractional SRLS problem, in which the computation of the worst-case residual is NP-complete.

The LFT approach provides a representation of the matrices containing uncertain data, preserving the structure of the uncertainties, and allows us to deal with the uncertain part of the system separately from the nominal system. The solution of the LFT problem is an NP-hard problem. El Ghaoui and Calafiore [10] have recently shown how the LFT problem can be recast into a robust semidefinite programming problem (SDP). The upper and lower confidence bounds can then be obtained by solving the SDP. 
The paper is organised as follows. In Section 2 we present state estimation algorithm and discuss the ill-conditioning of the Jacobian matrix. In Section 3 we obtain the linear fractional transformation of the linearised system equation. We discuss the method of El Ghaoui and Calafiore [10] in brief in Section 4 and use the LFT/SDP approach to formulate the uncertainty analysis problem for state estimation. The ideas are illustrated by a simple example of a twelve node water distribution network. Numerical results are obtained using MATLAB and ellipsoid-of-confidence bounds are reported, along with discussions, in Section 5. Finally, the conclusions are given in Section 6.

\section{State Estimation Algorithm}

When applied to a water distribution network a state estimator ideally provides the best possible information about the flows and pressures given all the available data. A water distribution system comprises a network of interconnected reservoirs, pipes, pumps and valves. The relationship between flow and pressure for each component is modeled using a non-linear equation of the form:

$$
q=f\left(\Delta p^{1 / \alpha}\right)
$$

where $q$ and $\Delta p$ refer to flow through and pressure across the element respectively. The function $f(\cdot)$ is nonlinear, smooth in the case of pipes and static valves but nonlinear and nonsmooth in the case of all other components. The exponent $\alpha$ is typically in the range $1.0<\alpha \leq 2.0$. An example of the flow in a pipe from node $i$ to node $j$ is the Hazen-Williams flow formula:

$$
\begin{aligned}
& q_{i j}=\Omega\left(h_{i}-h_{j}\right)\left|h_{i}-h_{j}\right|^{-0.46} \\
& \Omega=K_{u} C_{i j} D_{i j}^{2.63} L_{i j}^{-0.54}
\end{aligned}
$$

where $K_{u}$ is a units based constant, $D_{i j}$ is the diameter, $C_{i j}$ is called the Hazen-Williams constant (effectively a conductance term) and $L_{i j}$ is the length. The pressures are represented as heads (metres of water column) $h_{i}$ and $h_{j}$ at nodes $i$ and $j$ respectively.

The set of network equations are formulated to guarantee the satisfaction of Kirchoff's laws and can be set up as nodal equations e.g. [19] or loop equations as in [1].

A state estimator is based on this mathematical model of the network. Let $\tilde{z} \in \mathbb{R}^{m}$ denote the set of measurements, $\tilde{x} \in \mathbb{R}^{n}$ the vector of state variables, $g(\cdot)$ the nonlinear relation between the measured variables and the state variables, and $\epsilon \in \mathbb{R}^{m}$ the measurement error vector. A necessary but not sufficient condition for state estimation is $m>n$. Then a state estimator can be mathematically represented as:

$$
\tilde{z}=g(\tilde{x})+\epsilon
$$


where $\epsilon$ contains the measurement uncertainty and represents the discrepancy between the model and the real system. Traditionally, the following is believed to hold for $\epsilon$ :

$$
\begin{gathered}
\mathrm{E}(\epsilon)=0 \\
\mathrm{E}\left(\epsilon \epsilon^{T}\right)=R
\end{gathered}
$$

where $\mathrm{E}$ is the expectation operator, and the measurement error covariance matrix $R$ is such that:

$$
R_{i j}=\left\{\begin{array}{cc}
\sigma_{i}^{2} & i=j \\
0 & i \neq j .
\end{array}\right.
$$

The state estimates are said to be unbiassed if and only if (4), (5) and (6) hold true.

The solution to (3) can be found by minimising the WLS cost function:

$$
\min _{\tilde{x}} F(\tilde{x})=(\tilde{z}-g(\tilde{x}))^{T} R^{-1}(\tilde{z}-g(\tilde{x})) .
$$

We obtain the linearised form of the estimation problem from the first two terms of a Taylor series expansion of (3) about an iteration point $x_{0}$ :

$$
z=J x+\epsilon
$$

where $z=\tilde{z}-g\left(x_{0}\right)$, i.e the measurement residual vector; $x$ is the vector of incremental changes in the state, i.e. the difference between the next value of the state estimate and the current iterate, and $J \in \mathbb{R}^{m \times n}$, $m>n$, is the Jacobian of $g\left(x_{0}\right)$. The normal equations method calculates the state vector of incremental changes by solving the linear system:

$$
\left[J^{T} R^{-1} J\right] x=\left[J^{T} R^{-1}\right] z .
$$

Theoretically, a necessary and sufficient condition for the existence of a solution to (9) is that $J$ is of full rank. However, the conditioning of $J$ is often very poor which, combined with the uncertainties in both the parameters and measurements and the limited measurement redundancy, can lead to unrealistic results.

\section{Uncertainty Modelling for the State Estimation}

An uncertain parameter $p_{i}$ in the model can be written as $p_{i}=p_{i}^{o}+\Delta_{p_{i}}$ where $p_{i}^{o}$ is the nominal value of the parameter and $\Delta_{p_{i}}$ represents an unknown perturbation of the nominal value. This perturbation is constrained to an uncertainty set which represents the possible values of the perturbation. It is more straightforward to let all the different uncertainties belong to the same uncertainty set. This is accomplished by introducing a scaling factor to each perturbation such that $p_{i}=p_{i}^{o}+\delta_{i} \bar{p}_{i}$. The uncertainty set that $\delta_{i}$ belongs to can now be of any magnitude as $\bar{p}_{i}$ scales the uncertainty to the one corresponding to the perturbed parameter. The most obvious choice of the uncertainty set is when the magnitude is unity, i.e., for the real parameters, $\delta_{i} \in[-1,+1]$. 


\subsection{Linear Fractional Representation}

Taking into consideration the deterministic uncertainty in the network parameters $p_{i}$ appearing in the Jacobian matrix $J$, the measurement noise corrupted linearised system equation shown in (8), where $\epsilon$ is the stochastic uncertainty, can be written as:

$$
z=J\left(p_{1}, p_{2}, \ldots, p_{k}\right) x+\epsilon
$$

where, $p_{i}=p_{i}^{o}+\delta_{i} \bar{p}_{i}$ for $i=1,2, \ldots, k$, and $-1 \leq \delta_{i} \leq 1$.

Hence $\epsilon$ has now been redefined to represent just the measurement uncertainty (c.f. (8)) and, referring to (2), the parametric uncertainty (which can be due to any or all of the physical parameters) is contained in $\Omega$. For generality we let $p_{i}$ denote the uncertain parameters; $k$ being the number of uncertain parameters.

Rewriting (11) in terms of the nominal and the perturbed parameters we obtain:

$$
z=J_{o}\left(p_{1}^{o}, p_{2}^{o}, \ldots, p_{k}^{o}\right) x+\sum_{i=1}^{k} \delta_{i} J_{i}\left(\bar{p}_{i}\right) x+\epsilon
$$

where $J_{o}$ represents the nominal system. We can separate out the perturbations affecting $J_{o}$ and then collect them in a diagonal matrix $\Delta$ with the perturbations along its diagonal, as shown below.

Defining the following variables, of appropriate dimensions,

$$
\begin{array}{lll}
\xi_{o}=\beta \omega_{o}=\epsilon & \omega_{o}=\delta_{o} \xi_{o} & \text { such that } \beta=1 / \delta_{o} \\
\xi_{i}=J_{i}\left(\bar{p}_{i}\right) x & \omega_{i}=\delta_{i} \xi_{i} & \text { for } \quad i=1,2, \ldots, k
\end{array}
$$

where

$$
\delta_{o}, \delta_{i} \in[-1,+1]
$$

equation (12) becomes:

$$
z=J_{o}\left(p_{1}^{o}, p_{2}^{o}, \ldots, p_{k}^{o}\right) x+\sum_{i=1}^{k} \omega_{i}+\beta \omega_{o},
$$

and collecting equations (13) and (15) in a matrix form we have:

$$
\left[\begin{array}{l}
\xi \\
z
\end{array}\right]=M\left[\begin{array}{l}
\omega \\
x
\end{array}\right]=\left[\begin{array}{ll}
M_{11} & M_{12} \\
M_{21} & M_{22}
\end{array}\right]\left[\begin{array}{l}
\omega \\
x
\end{array}\right]
$$

and

$$
\omega=\Delta \xi \text { for some } \Delta \in \Delta \text {, where } \boldsymbol{\Delta}=\left\{\Delta \mid\|\Delta\|_{\infty} \leq 1\right\},
$$

together with,

$$
\begin{aligned}
\xi & =\left[\begin{array}{lllll}
\xi_{o}^{T} & \xi_{1}^{T} & \xi_{2}^{T} & \ldots & \xi_{k}^{T}
\end{array}\right]^{T} \text { and } \\
\omega & =\left[\begin{array}{lllll}
\omega_{o}^{T} & \omega_{1}^{T} & \omega_{2}^{T} & \ldots & \omega_{k}^{T}
\end{array}\right]^{T}
\end{aligned}
$$


and the block diagonal matrix $\Delta$ given by:

$$
\Delta=\operatorname{diag}\left(\delta_{o} I_{m}, \delta_{1} I_{m}, \delta_{2} I_{m}, \ldots, \delta_{k} I_{m}\right)
$$

where $I_{m}$ denotes an identity matrix of dimension $m \times m$.

From (16) and (17) we obtain the following equation:

$$
z=\mathcal{F}_{u}(M, \Delta) x, \quad \forall \Delta \in \Delta=\left\{\Delta \mid\|\Delta\|_{\infty} \leq 1\right\}
$$

where the interconnection matrix $\mathcal{F}_{u}(M, \Delta)$ is given by:

$$
\mathcal{F}_{u}(M, \Delta)=M_{22}+M_{21} \Delta\left(I-M_{11} \Delta\right)^{-1} M_{12}
$$

The matrix in (21) is known as the upper linear fractional transformation (LFT) of the uncertain system represented by equations (16) and (17). The physical meaning of the LFT shown in Figure 1, and defined by (21), is that it represents the closed-loop transfer matrix from $x$ to $z$ where $M$ is the plant matrix. The LFT $\mathcal{F}_{u}(M, \Delta)$ has a nominal mapping $M_{22}$ and is perturbed by $\Delta$; the mappings $M_{11}, M_{12}$, and $M_{21}$ reflect a priori knowledge as to how the perturbation affects the nominal map $M_{22}$ [23]. In a similar fashion a lower LFT can be defined. More on LFTs and their interesting properties can be found in [7, 23].

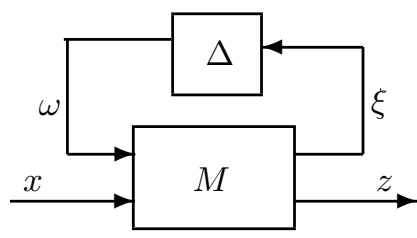

Figure 1: LFT representation of the uncertain system given by equations (16) \& (17).

The main advantage of an LFT representation is that it separates the uncertainties from the nominal system. In robust control engineering terminology this principle is said to "pull out the uncertainties" [23].

The LFT introduced above is said to be well-posed if the determinant $\operatorname{det}\left(I-M_{11} \Delta\right) \neq 0$. But it may not necessarily be well-posed over $\boldsymbol{\Delta}$, and it might happen that $\operatorname{det}\left(I-M_{11} \Delta\right)=0$ for some $\Delta \in \boldsymbol{\Delta}$. Checking well-posedness is the equivalent of solving (20), which is an NP-hard problem, known in robust control theory as the $\mu$ or structured singular value (SSV) analysis problem, addressed in [7].

\section{Uncertainty Analysis using SDP Approach}

We now follow the 'robust semidefinite programming' (SDP) approach of El Ghaoui and Calafiore [10] to solve (20). 
Under the assumption that the LFT is well-posed, we can approximate the uncertain input-output relation by a set of quadratic constraints. In conjunction with the $\mathcal{S}$-procedure for linear matrix inequalities (LMI) we obtain the ellipsoids of confidence, of minimal size in a certain geometrical sense, by recursively solving a semidefinite programming problem.

The basic problem at hand is to compute an ellipsoid of confidence for the state estimates $x$, where:

$$
\mathcal{X}=\left\{x \mid z=\mathcal{F}_{u}(M, \Delta) x \text { for some } \Delta \in \Delta\right\} .
$$

Let us model an ellipsoid that we seek as a confidence region for the state estimates as:

$$
\mathcal{E}=\left\{x \mid P \succeq(x-\hat{x})(x-\hat{x})^{T}\right\}
$$

where $\hat{x}$ denotes the centre of the ellipsoid and $P=P^{T} \succeq 0$ is a symmetric positive-definite matrix that determines the shape of the ellipsoid. The size of the ellipsoid is measured by the sum of the squares of the semi-axis length of the ellipsoid i.e. the Trace of $P$, denoted by $\operatorname{Tr} P$.

We seek to minimise the size of the ellipsoid $\mathcal{E}$ subject to (22) for every $\Delta \in \boldsymbol{\Delta}$. Our problem, therefore, reduces to the following robust semidefinite program:

$$
\min _{\hat{x}, P} \operatorname{Tr} P
$$

such that $P \succeq 0$, and

$$
\left[\begin{array}{cc}
P & \hat{x}-\mathcal{F}_{u}(M, \Delta)^{\dagger} z \\
\left(\hat{x}-\mathcal{F}_{u}(M, \Delta)^{\dagger} z\right)^{T} & I
\end{array}\right] \succeq 0
$$

for every $\Delta \in \Delta=\left\{\Delta \mid\|\Delta\|_{\infty} \leq 1\right\}$

where ${ }^{\dagger}$ denotes the Moore-Penrose pseudo-inverse of the over-determined rectangular matrix $\mathcal{F}_{u}(M, \Delta)$.

Intervals of confidence for $x$ (complementary bounds for the point state estimate) can be obtained by projecting the ellipsoid of confidence on the coordinate axes:

$$
x_{j}^{l}=\hat{x}_{j}-\sqrt{P_{j j}} \text { and } x_{j}^{u}=\hat{x}_{j}+\sqrt{P_{j j}}
$$

where $j$ represents the $j$ th coordinate axes or the $j$ th component of the state vector $x$, and $x_{j}^{u}$ and $x_{j}^{l}$ denote the upper and lower ellipsoid-of-confidence bounds on the state estimates, respectively, such that:

$$
x_{j}^{l} \leq x_{j} \leq x_{j}^{u}
$$

To obtain the ellipsoid-of-confidence bounds we solve the semidefinite programming problem (24) subject to (25). The linear matrix inequality (25) is represented using MATLAB and the semidefinite program is solved using the LMI control toolbox available with the MATLAB package. The optimisation function for solving the SDP in MATLAB uses the interior point algorithm for SDP developed by Nesterov and Nemirovski $[17,9]$. 


\section{Illustration by an Example}

The ideas discussed in this paper are applied to a small water distribution network to illustrate the concept. For the network shown in Figure 2 the network topology and parameter values are given in Table 1. In this test network there are two fixed head nodes, 13 and 14, which represent the reservoirs. The water network is simulated using the mathematical package MATLAB, which solves an exact set of nonlinear flow and mass balance equations and thus provides a steady state flow model.

Any choice of meter coverage is arbitrary. In the example, a uniform coverage has been chosen in that each node has exactly one pressure transducer. In addition, each node has a demand, $d_{i}$, representing the supply to consumers. Thus $m=24$ and $n=12$. In strategic water supply networks metering redundancy levels are approaching this richness. Furthermore, power systems often have redundancy levels $m / n \geq 2$. In water distribution networks, however, the redundancy level could be much lower.

To simulate real measurements we perturb the simulated heads and nodal demands by choosing from a Gaussian distribution of random values where $\pm 3 \sigma \equiv \pm 25 \%$. Furthermore, the parametric uncertainties are represented as a flat distribution of $C$ values bounded by $\pm 10 \%$ of the nominal value. Example values are shown in Table 1 together with the remaining network parameters.

In order to compare the results with previous methods, a conventional WLS state estimation problem (which can only deal with measurement uncertainty) is run on the data. Alongside this we present the results from the LFT/SDP approach with uncertainty in both the measurements and the parameters. The confidence bounds are calculated using equations in (26) and are presented, together with the WLS state estimates, in Table 2.

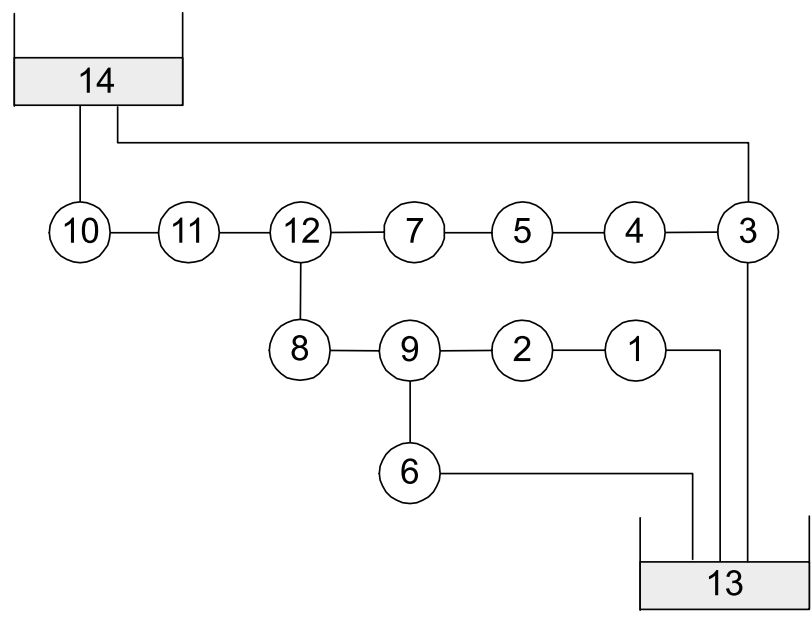

Figure 2: Example water network: 12 nodes. 
Table 1: Example water network: data.

\begin{tabular}{|c|c|c|c|c|c|c|c|c|c|}
\hline \multicolumn{7}{|c|}{ Network parameters } & \multicolumn{3}{|c|}{ Measurements } \\
\hline \multirow{2}{*}{\multicolumn{3}{|c|}{ Link From To }} & ength & Diameter & $C$ & $\Omega$ & Node & emand & Fixed \\
\hline & & & $(\mathrm{m})$ & $(\mathrm{mm})$ & values & (c.f. (2)) & & $(\mathrm{l} / \mathrm{s})$ & heads $(\mathrm{m})$ \\
\hline 1 & 1 & 13 & 460 & 250 & 90 & 23.7793 & 1 & 5 & \\
\hline 2 & 2 & 1 & 400 & 250 & 90 & 25.6434 & 2 & 5 & \\
\hline 3 & 9 & 2 & 320 & 250 & 100 & 32.1414 & 3 & 5 & \\
\hline 4 & 3 & 13 & 140 & 250 & 85 & 42.6928 & 4 & 5 & \\
\hline 5 & 4 & 3 & 200 & 250 & 110 & 45.5703 & 5 & 10 & \\
\hline 6 & 5 & 4 & 300 & 250 & 90 & 29.9531 & 6 & 5 & \\
\hline 7 & 6 & 13 & 500 & 250 & 100 & 25.2581 & 7 & 5 & \\
\hline 8 & 9 & 6 & 620 & 250 & 90 & 20.2393 & 8 & 5 & \\
\hline 9 & 8 & 9 & 285 & 250 & 120 & 41.0592 & 9 & 10 & \\
\hline 10 & 14 & 3 & 460 & 250 & 120 & 31.7057 & 10 & 5 & \\
\hline 11 & 14 & 10 & 160 & 250 & 130 & 60.7524 & 11 & 5 & \\
\hline 12 & 10 & 11 & 170 & 250 & 75 & 33.9206 & 12 & 5 & \\
\hline 13 & 11 & 12 & 145 & 250 & 80 & 39.4272 & 13 & & 100 \\
\hline 14 & 12 & 8 & 340 & 250 & 75 & 23.3296 & 14 & & 120 \\
\hline 15 & 12 & 7 & 320 & 250 & 60 & 19.2848 & & & \\
\hline 16 & 7 & 5 & 130 & 250 & 90 & 47.0499 & & & \\
\hline
\end{tabular}

\subsection{Discussions}

In reality, water, power and gas distribution networks are of a much higher dimension, e.g. 500 to 1000 times larger than the simple example shown here. Nevertheless, some interesting observations can be made.

Within the LFT/SDP algorithm uncertainty values in $\Delta$ (measurement and parameter) are computed in the range $[-1,1]$ and the input measurement data is also corrupted by noise (as it is in the WLS estimator), on the other hand the WLS parameter values are considered exact. Therefore, the LFT/SDP approach highlights the likely effect of parametric and measurement uncertainty on flow and pressure calculations, which is an important adjunct to the estimates themselves.

When equations (4) - (6) are satisfied (which also implies that there is no bad data), a weighted least squares state estimator is termed 'BLUE' (best linear unbiassed estimator). However, due to the large amount 
of measurement noise present (e.g. 25\%), we see fairly large discrepancies between the simulated values and the WLS estimates. Interestingly, the values of the centre of the ellipsoid are closer to the simulated values in every instance except those for the nodes directly connected to the reservoirs. Studies into this result for larger, more realistic networks, are on-going and will be the subject of a subsequent publication. The LFT/SDP algorithm was developed to compute the confidence bounds on the state estimates and whilst the centre of the ellipsoid could be interpreted as an estimate, it is not guaranteed optimal. According to [18], the TLS method is sensitive to small perturbations and the method used, which is based on singular value decomposition (SVD), does not preserve the structure. Furthermore, it yields only approximate solutions. To resolve this, various regularisation methods have been developed to improve the conditioning of the matrices but in so doing they introduce a small amount of bias to the problem. Also the choice of the regularisation parameter is very much problem dependent. This point also applies to the $\sigma_{i}$ values in WLS estimation, which are required a priori but which are rarely known exactly. In comparison, the LFT uses a more or less arbitrary range of values to define the original confidence in the parameters and measurements.

The set-membership method can be used to deal with $A x \approx b$ problems with $A, b$ uncertain but SBSE is restricted to additive uncertainty whereas the LFT approach could be extended to deal with multiplicative uncertainty. Furthermore, SBSE leads to a non-convex programming problem [4] and therefore a brute force method has to be used to transform the non-convex program into (say) an LP. However, the LFT approach leads directly to a convex optimisation problem.

The provision of bounds as in SBSE methods, which define a region in state-space, is less helpful than the computation of an ellipsoid of confidence, the principal axes of which could be successively reduced to produce an optimal point or at worst a line in the degenerate case. However, as we can see above, using the centre directly may well be sufficient.

\section{Conclusions}

For the state estimation to produce accurate results it is important to know the reliability of the measurements which are contaminated by noise and the uncertain parameters appearing in the system equations. In this paper we have shown how the LFT/SDP based formulation can be used to obtain the confidence bounds on the state estimates in the context of a water distribution network. The following conclusions can be drawn:

- Whereas the WLS methods produce point state estimates in the presence of only measurement noise, the proposed LFT/SDP approach produces confidence-of-ellipsoid bounds in the presence of measurement noise as well as parametric uncertainty. The centre of the ellipsoid is a robust estimate and could be interpreted as a best point estimate. 
Table 2: Comparison of simulated states, estimated states, and ellipsoid-of-confidence bounds for the state estimates.

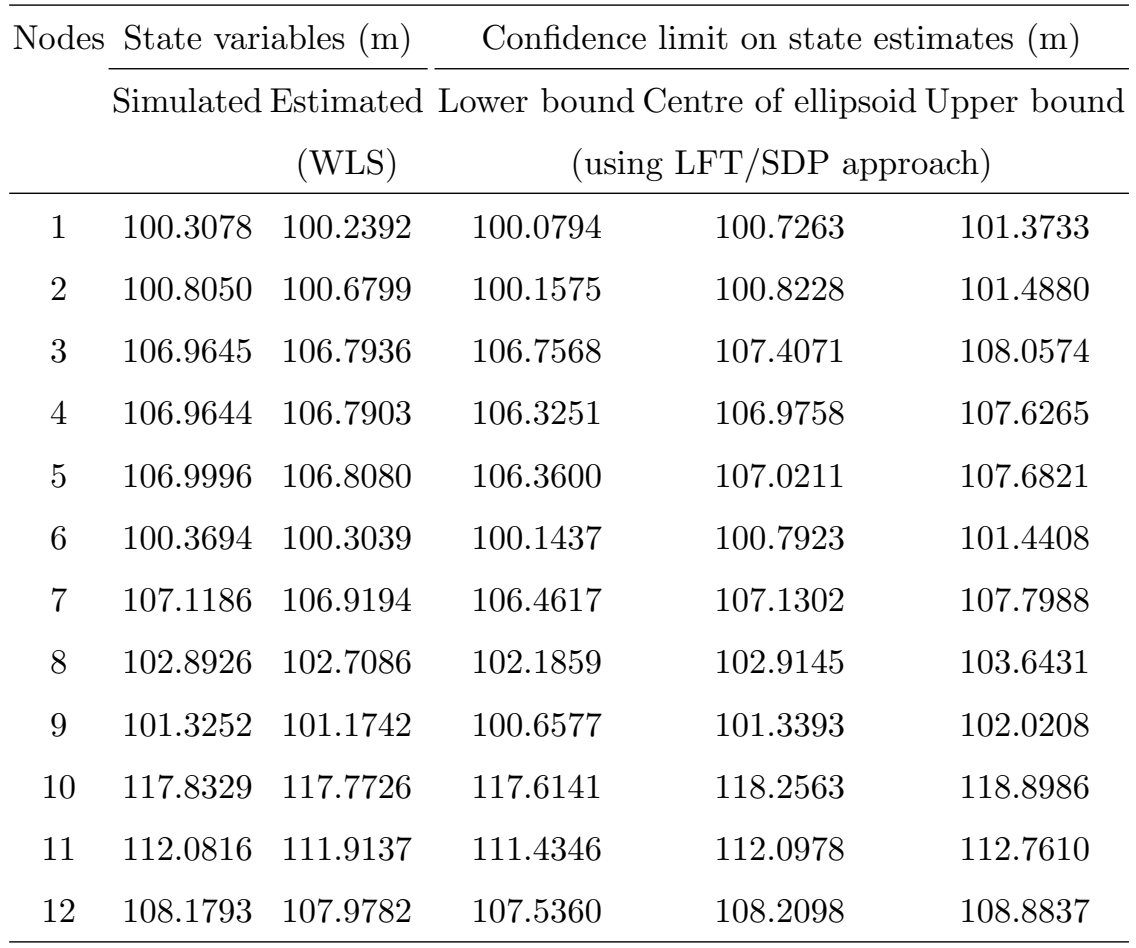

- An LFT formulation provides a unified approach to model measurement noise (Gaussian) as well as parametric uncertainty (deterministic) in the same framework, and reduces to a SDP (a convex optimisation problem). This is an advantage of the proposed method over the SBSE approach which models the measurement noise (as well as uncertainty) as a flat distribution.

- We have shown that the LFT/SDP approach is an effective method for solving the problem of uncertainties on both sides of $A x \approx b$.

- By providing bounds on the estimates one can infer the quality of the metering configuration and determine whether the installation of new meters would be desirable.

- Although couched in the context of water networks, the approach could be used for a wide range of systems having models of a similar type.

\section{Acknowledgements}

This work is an on going research project supported by the Engineering and Physical Sciences Research Council, (E.P.S.R.C.), U.K., Thames Water Utilities Ltd., South West Water Services Ltd., and RPS Services 
Ltd. The authors are indebted to these bodies for their financial support.

\section{References}

[1] J. H. Andersen, R. S. Powell, Simulation of Water Networks Containing Controlling Elements', in: ASCE Journal of Water Resources Planning and Management, vol 125, no. 3, 1999, pp. 162-169 .

[2] A. Bargiela, G. Hainsworth, Pressure and Flow Uncertainty in Water Systems, in: ASCE Journal of Water Resources Planning and Management, vol. 115, no. 2, 1989, pp. 212-229.

[3] S. Boyd, L. El Ghaoui, E. Feron, V. Balakrishnan, Linear Matrix Inequalities in Systems and Control Theory, SIAM Books, Philadelphia, 1994.

[4] M. A. Brdys, K. Chen, Joint State and Parameter Estimation of Dynamic Water Supply Systems Under Bounded Uncertainty, in: Integrated Computer Applications in Water Supply, B. Coulbeck (ed.), vol.1, (Research Studies Press Ltd.), 1993, pp. 335-353.

[5] S. Chandrasekaran, G. H. Golub, M. Gu, A. H. Sayed, Parameter Estimation in the Presence of Bounded Data Uncertainties, in: SIAM J. Matrix Anal. Appl., vol. 19, no. 1, January 1998, pp. 235-252.

[6] J. C. Doyle, Lecture Notes on Advances in Multivariable Control, in: ONR/Honeywell Workshop, Minneapolis, October 1984.

[7] J. C. Doyle, A. Packard, K. Zhou, Review of LFTs, LMIs, and $\mu$, in: Proc. of 30th. CDC, Brighton, England, 1991, pp. 1227-1232.

[8] B. Gabrys, A. Bargiela, Integrated Neural Based System for State Estimation and Confidence Limit Analysis in Water Networks, Proceedings of European Simulation Symposium ESS'96, (Genoa), ISBN 1-565555-099-4 vol.2, 1996, pp. 398-402.

[9] P. Gahinet, A. Nemirovski, A. J. Laub, M. Chilali, LMI Control Toolbox User's Guide, The Mathworks Partners Series, The Math Works Inc., 1995.

[10] L. El Ghaoui, G. Calafiore, Worst-Case State Prediction under Structured Uncertainty, in: Proc. of American Control Conference, San Diego, California, June 1999, pp. 3402-3406.

[11] L. El Ghaoui, H. Lebret, Robust Solutions to Least Squares Problems with Uncertain Data, in: SIAM J. Matrix Anal. Appl., vol. 18, no. 4, October 1997, pp. 1035-1064.

[12] G. H. Golub, C. F. Van Loan, Matrix Computations, The Johns Hopkins University Press Books, Baltimore, third ed., 1997. 
[13] E. Handshin, F. C. Schweppe, J. Kohloas, A. Fletcher, Bad Data Analysis for Power System State Estimation, in: IEEE, PES, Summer Meeting \& Energy Resources, Anaheim, California, 1974.

[14] M. R. Irving, R. C. Owen, M. J. H. Sterling, Power System State Estimation using Linear Programming, in: IEE Proceedings, vol. 125-9, 1978, pp. 879-885.

[15] L. Mili, T. Van Cutsem, M. Ribbens-Pavella, Hypothesis Testing Identification: A New Method for Bad Data Analysis in Power Systems State Estimation, in: IEEE Transactions PAS, vol. 103, no.11, 1984, pp. 3239-3252.

[16] A. Monticelli, F. F. Wu, Y. Masong, Multiple Bad Data Identification for State Estimation by Orthogonal Transformations, in: IEEE Transactions PAS, vol. 104, no.12, 1985, pp. 3460-3468.

[17] Yu Nesterov, A. Nemirovski, Interior Point Polynomial Methods in Convex Programming: Theory and Applications, SIAM Books, Philadelphia, 1994.

[18] J. B. Rosen, H. Park, J. Glick, Total Least Norm Formulation and Solution for Structured Problems, in: SIAM J. Matrix Anal. Appl., vol. 17, no. 1, January 1999, pp. 110-126.

[19] R. S. Powell, On-Line Monitoring for Operational Control of Water Distribution Networks, Ph.D. thesis, the University of Durham, U.K., 1992.

[20] R. S. Powell, M. R. Irving, M. J. H. Sterling, A Comparison of Three Real-Time State Estimation Methods for On-Line Monitoring of Water Distribution Systems, in: Computer Applications in Water Supply, B. Coulbeck (ed.), vol. 1, (Research Studies Press Ltd.), 1988, pp. 333-348.

[21] M. J. H. Sterling, A. Bargiela, Minimum Norm State Estimation for Computer Control of Water Distribution Systems, in: IEE Proceedings, part D., vol. 131, no.2, 1984, pp. 57-63.

[22] N. Xiang, S. Wang, E. Yu, A New Approach for Detection and Identification of Multiple Bad Data in Power System State Estimation, in: IEEE Transactions PAS, vol. 101, no.2, 1982, pp. 454-462.

[23] K. Zhou, J. C. Doyle, K. Glover, Robust and Optimal Control, Prentice Hall Books, Upper Saddle River, New Jersey, 1996. 